\section{COMPREHENSIVE MULTI-OMICS META-ANALYSIS OF PANCREATIC CANCER MOUSE MODELS AND HUMAN PDAC DATA SETS IDENTIFIES UNIQUE CANCER- ASSOCIATED FIBROBLAST SUBSETS}

${ }^{1}$ Candace Wai Sze Lei*, 'John Holt, 'Brianna Flynn, ${ }^{2}$ Richard Barrett, 'Pratha Budhani, ${ }^{1}$ Mohanapriya Kamalakannan, ${ }^{1}$ Ruby Wasti, 'Lucinda Thiede, ${ }^{1} J o s h u a$ Tagore, 'Jessica Potts, 'Jacqueline Larouche, 'Marie Marcher, 'Xiaoyun Liao, 'Sarah O'Brien, ${ }^{1}$ Abhishek Kashyap, 'Jeanine Pignatelli, ${ }^{1}$ Kang Liu, ${ }^{1}$ Joseph Tumang, ${ }^{1}$ Emily Corse, ${ }^{3}$ Ben Stanger, ${ }^{2}$ Ellen Pure, ${ }^{1}$ Varenka Rodriguez DiBlasi. 'Boehringer Ingelheim, Ridgefield, CT, United States; ${ }^{2}$ University of Pennsylvania School of Veterinary Medicine, Philadelphia, PA, United States; ${ }^{3}$ University of Pennsylvania Perelman School of Medicine, Philadelphia, $P A$, United States

Background Pancreatic ductal adenocarcinoma (PDAC) is resistant to many available therapies including immunotherapy because of its highly complex tumor microenvironment (TME). PDAC TME consists of a significant proportion of stromal cells, such as endothelial cells, perivascular cells, and cancer-associated fibroblasts (CAFs). Recent work indicates how CAFs can orchestrate the crosstalk cancer and immune cells, and contribute to many aspects of tumor progression, including angiogenesis, senescence, and inflammation. Recent studies based on scRNA-seq have increased understanding of CAF heterogeneity in PDAC in both human and genetically engineered mouse models (GEMMs) is of high interest. To understand the translatability of GEMMs in the setting of PDAC, we conducted a thorough scRNA-seq meta-analysis on CAFs across GEMMs and PDAC human samples. Hereafter, we characterized CAFs multi-dimensionally based on transcriptional, chromatin accessibility, and spatial profiles. Finally, we suggested certain transcription factors may be regulatory drivers of heterogeneous CAF phenotypes in both human and GEMMs.

Methods We collected publicly available and internally generated scRNA-seq data of PDAC CAFs from human and mouse. After dataset alignment and label transfer, we conducted differential expression analysis across CAF subsets to characterize myofibroblasts (myCAFs) and other CAF subsets of interest. Bioinformatically, we further interrogated CAF heterogeneity in terms of regulatory potential of transcription factors, gene set enrichment, and functional state transition. Complemented by epigenomic assessment, we investigated chromatin accessibility and transcription factor binding availability on the single-cell level. Finally, to investigate the TME organization and spatial neighborhood of cell-to-cell interaction, we explored potential functional differences across location and transcriptional changes of CAF subsets by spatial transcriptomics.

Results We found that myofibroblasts (myCAFs) make up a substantial proportion of the CAF population, in both human and mouse TME. In a combination of transcriptional profiling, chromatin accessibility assessment, and spatial transcriptomics, we elucidated potential functional and phenotypic differences within myCAF population and compared to other CAF subsets in the TME. While myofibroblasts are traditionally described as matrix remodeling related, heterogeneity in myofibroblasts may suggest additional roles played by this specific subset. In addition, CAFs in human and mouse share similarities, in terms of transcriptional profiles and phenotypes. The use of GEMMs facilitates our understanding of CAF heterogenous behavior and phenotypes in the PDAC TME.

Conclusions Here, we presented a comprehensive overview of CAF heterogeneity in mouse PDAC models and human datasets. Our observations highlight molecular differences in CAFs, which facilitates our understanding on PDAC stromal microenvironment and translatability in GEMMs in imitating human TME.

http://dx.doi.org/10.1136/jitc-2021-SITC2021.902 